\title{
A preliminary study on nasalization and phonation
}

\section{The case of Bai}

\author{
Xuan Li and Feng Wang \\ Chinese Academy of Social Sciences $\mid$ Peking University
}

Although it is widely acknowledged that different speech processes may interact with each other, the way that nasalization affects phonation remains poorly understood. This paper explores the relationship between nasalization and phonation, by analyzing the phonetic cues of the tense/lax distinction both in nasalization and non-nasalization in the Bai language. The data for discussion is from two Bai dialects, Chengbei and Jinhua, which have a tense/lax distinction in both nasalized and non-nasalized syllables. Three phonation parameters - fundamental frequency (F0), open quotient (OQ), and speed quotient (SQ) - are extracted from EGG signals for analysis. It is found that the influence of nasalization on phonation varies with the tone contours. As for the level tones, the role of phonation manner in tone distinction is not evident in nasalization in that tense tones can be distinguished from lax tones only by pitch. However, in nonnasalization, phonation manner plays an indispensable role in tone distinction, in that the contrast between tense and lax tones are reflected not only on F0 but also on OQ and SQ. Moreover, non-nasalized tense tones are more likely to be accompanied by non-modal phonation that is characterized by a significantly higher SQ. In terms of articulatory explanation, high SQ in non-modal phonation is the result of the vibration of tightened vocal folds, and the tension of vocal folds is caused by raising the soft palate in non-nasalization. As for the falling tones, the role of phonation manner in tone distinction is more salient in nasalization than in non-nasalization in Chengbei Bai, but it is not attested in Jinhua Bai. This study shows that the interaction between nasalization and phonation in Bai can be revealed in the analysis of phonation parameters, i.e. F0, OQ, and SQ.

Keywords: nasalization, phonation, Bai, fundamental frequency, open quotient, speed quotient 


\section{Literature review}

\subsection{Research into phonation in Bai}

The tense/lax distinction of syllables in the Bai language was noticed early and used to be treated as the feature of vowels as in the Yi language. Xu \& Zhao (1984) hold a similar view, claiming that the tense feature in Bai is associated with vowels. Besides, they also argue that such distinction in Bai is related to tones, in that vowels are tenser when bearing certain tones, i.e. $/ 42 /, / 21 /$, or $/ 44 /$ in most dialects. Therefore, for convenience, the tense feature is combined with the pitch as tone category in the phonological description. In Chao (1980[1930])'s devise, for all tone-letters, a simplified pitch contour is attached to the left of a vertical reference line, while in Xu \& Zhao, the contour of lax tones is usually marked on the left, and that of tense tones on the right (e.g. $\downarrow$ indicates a lax level tone, while $\vdash$ indicates a tense level tone). Later studies all follow Xu \& Zhao, considering tense/lax distinction as the feature of tone categories in Bai. In Dai \& Zhao's (2009) description of Zhaozhuang Bai, tones /42/ and /21/ are tenser and accompanied by a glottal stop. In the reference grammar of Kangfu Bai (Zhao 2010), tone /42/, /21/, and $/ 44 /$ are regarded as tense tones. The tense/lax difference is proposed as a phonological property in Bai, but what is its phonetic basis?

Maddieson \& Ladefoged (1985) investigated the nature of tense/lax tones in descriptions of four languages: Hani, Yi, Jingpo, and Wa. Acoustic and aerodynamic properties of the vowels were examined in these languages. It is found that the prime distinction between tense and lax contrasts is the phonation type, and other characteristics are subordinate. In the 1990s, the studies of phonation types received further attention in China. Scholars, from Voice Laboratory of Chinese Academy of Social Sciences, conducted a series of studies on phonation types in different ethnic languages in China by applying experimental phonetics or acoustic approaches. Their research covers various phonation types, providing a whole picture of phonation types in languages of China (Bao \& Lü 1992; Li 1992; Kong 1996).

At the same time, much research in experimental phonetics studied the nature of the tense and lax contrasts in Bai. Li Shaoni and Edmondson (a.k.a. 艾傑瑞 Ài Jiéruì) investigated the tense tones of Bai by different instruments, such as miniature laryngoscopes. They claimed that instead of pitch, it is voice quality, such as creakiness, that contributes to the tonal contrasts in Jianchuan Bai ( $\mathrm{Li} \& \mathrm{Ai}$ 1990; Li 1992; Edmondson \& Li 1994; Edmondson et al. 2000; Edmondson et al. 2001). Benner (2009) applied Esling (2005)'s model of the vocal tract in her study and demonstrated that syllabic utterances of the Bai infants (by the end of the first year) are mostly constricted or dynamic, which reflects the use of the laryn- 
geal voice quality in Bai. Through analyzing voice waves and broad-band spectrograms, Yang \& Gao (2016) also observed the phonation type existing in the Bai tonal system. Tone ${ }^{\star} 1 \mathrm{~b}$ of Proto-Bai has developed into a low falling tone in some dialects but into a rising tone in still others. If it is a falling tone, it has a creaky phonation; if it is a rising tone, it is accompanied by a glottal stop.

Based on studies of Hani, Miao, Yi, Zaiwa, and Jingpo, Kong (2001) proposed a phonation-based theoretical framework of the phonetics. In this framework, the production of sound mainly consists of two parts. One part is phonation: the airflow exits the lungs, and goes through the vocal cords, making the vocal cords vibrate to produce the voice. Another part is articulation: the voice resonates with different resonance cavities and forms diverse sounds. The production of tone belongs to phonation, and tone is further decomposed into Tiaoshi (調時) 'temporal tone' and Tiaosheng (調聲) 'phonation tone.' The temporal tone represents the speed of the vocal folds vibrating in the temporal domain, which can be measured by the fundamental frequency (F0) value and F0 contour, while the phonation tone represents the manner of the vocal folds vibrating, which have attracted various methods of phonation research (Kong 2001:23-52).

In Kong's (2001) framework, further studies have been done on the tense feature and phonation types of Bai (Wang 2015; Li \& Wang 2016; Li 2017; Liu et al. 2019). These studies suggest that the distinction between tense and lax in Bai is the contrast of phonation types sometimes, and phonation manner plays a role in distinguishing tones in most cases. In addition, the distinctive phonetic features of tense and lax are found to vary both with respect to individuals and different tones.

\subsection{Phonation and nasalization}

Matisoff (1975) has brought up a concept called rhinoglottophilia, which refers to an affinity between the feature of nasality and the articulatory involvement of the glottis. Rhinoglottophilia is confirmed when vowels become nasalized in the environment of "laryngeals" in a variety of genetically unrelated languages. The close relationship between the lowering of the velum and the articulation of laryngeal sounds is supported by both synchronic and diachronic evidence in Matisoff (1975). Moreover, the articulatory settings of rhinoglottophilia have been comprehensively investigated in Ohala $(1971 ; 1972)$. Therefore, it is possible that laryngeals induce nasalization. Once nasalized, the phonetic attributes of vowels will be changed accordingly. Fant (1960:148-161) reviewed the research on acoustic characteristics of nasalization and pointed out that amplitude of the first formant is reduced in nasalization due to an increase in its bandwidth and the rise in the first formant frequency. By analyzing the properties of nasalized and 
non-nasalized vowel /a/, Singhal \& Das (2013) confirmed the significant difference between the waveforms of nasalized and non-nasalized vowels, and they further concluded that in terms of pitch period, nasalized /a/ has a cyclical nature and steeper/taller peaks which can be attributed to the combination of oral and nasal resonances. It can be seen that, on the one hand, nasalization is relatively independent in the process of speech production, but on the other hand, nasalization is not absolutely independent because it can be induced by other speech processes and is accompanied by a change in phonetic features.

Furthermore, Garellek et al. (2016) examined the breathy voice quality of nasal consonants and vowels. It demonstrated that nasal consonants are often breathier than laterals, as are vowels following nasals when compared to vowels following oral consonants. Breathy is known as a typical phonation type, thus the research of Garellek et al. (2016) also suggested that phonation is indeed influenced by nasalization.

Both the distinction of tense/lax and that of nasalized and non-nasalized vowels exist in some Bai dialects. For this reason, two of these Bai dialects will be studied in this paper to explore whether nasalization affects the phonation process, and - if so - how.

\section{Introduction of two Bai dialects}

Most Bai dialects in Dali City have no nasal endings or nasalized vowels, e.g. Meiba Bai (Li 2017), Longfeng Bai (Gong et al. 2018); but some Bai dialects out of Dali City do have nasalized vowels, e.g. Jinhua Bai (Xu \& Zhao 1984), Kangfu Bai (Zhao 2010), and Chengbei Bai.

This study is primarily based on the data from a series of field investigation of Chengbei Bai by the authors from 2016 to 2017, supplemented by Jinhua Bai material collected by Wang (2015). The phonological system of Jinhua Bai is described in Wang (2015), and that of Chengbei Bai is introduced as follows.

Chengbei Bai has twenty-two initials and thirty-nine finals. Fourteen finals are nasalized. It has seven tones, transcribed as T1 to T7 shown in Table 1. Three of seven tones - T2, T6, and T7 - are marked as tense tones. The tone value is designated in accordance with the five-tone scale devised by Chao (1980[1930]). It records the relative height of dissimilar tones in Chengbei Bai. 
Table 1. The tonal system of Chengbei Bai

\begin{tabular}{llcl}
\hline Tone name $^{*}$ & Tone quality & Tone value & Example \\
\hline T1 & Lax & 33 & kəu1 'bronze' \\
T2 & Tense & 42 & pe2 'white' \\
T3 & Lax & 31 & pe3 'ill' \\
T4 & Lax & 55 & kəu4 'hook ${ }_{\text {verb }}$, \\
T5 & Lax & 35 & p 5 'crack \\
T6 & ' \\
T7 & Tense & 44 & kəu6 'save' \\
\hline
\end{tabular}

* Tone names used by Xu \& Zhao (1984) in accord with the order of tones in the Bai pronunciation of Chinese tone categories “Yin 陰, Yang 陽, Shang上, Qu 去, and Ru 入”.

\section{Research methods}

3.1 Speakers and recording samples

\subsubsection{Chengbei Bai}

This study records the EGG signals of eight Bai speakers of Chengbei Village, Xiangyun County, Yunnan Province. Table 2 shows the basic information of eight speakers, including four males and four females. The letter before number presents gender with $\mathrm{M}$ for male, and $\mathrm{F}$ for female.

Table 2. The speakers of Chengbei Bai

\begin{tabular}{llcllc}
\hline Name & No. & Age & Name & No. & Age \\
\hline YM & F1 & 16 & YZC & M1 & 28 \\
YQ & F2 & 20 & YLK & M2 & 37 \\
DHY & F3 & 50 & YLZ & M3 & 42 \\
YXZ & F4 & 53 & YYZ & M4 & 48 \\
\hline
\end{tabular}

All the samples consist of initial stop consonants (very few syllables with zero initials), as shown in Table 3. In the following discussion, tone value combined with "T" (which means "tone") is used to present tones, e.g. T33 indicates lax T1 in Table 1.

"Tense" and "lax" are a pair of relative and interdependent concepts. When the tense tones are analyzed in Bai, the lax tones are also required to be studied for comparison. Therefore, six tones out of seven in Chengbei Bai will be discussed in this study. Three of them are tense tones, which are T44, T42, and T21, and 
their corresponding lax tones are T33, T31. In terms of falling tones, T31 is laxer than both T21 and T42, so it is compared to two tense tones respectively then.

Table 3. Recording samples

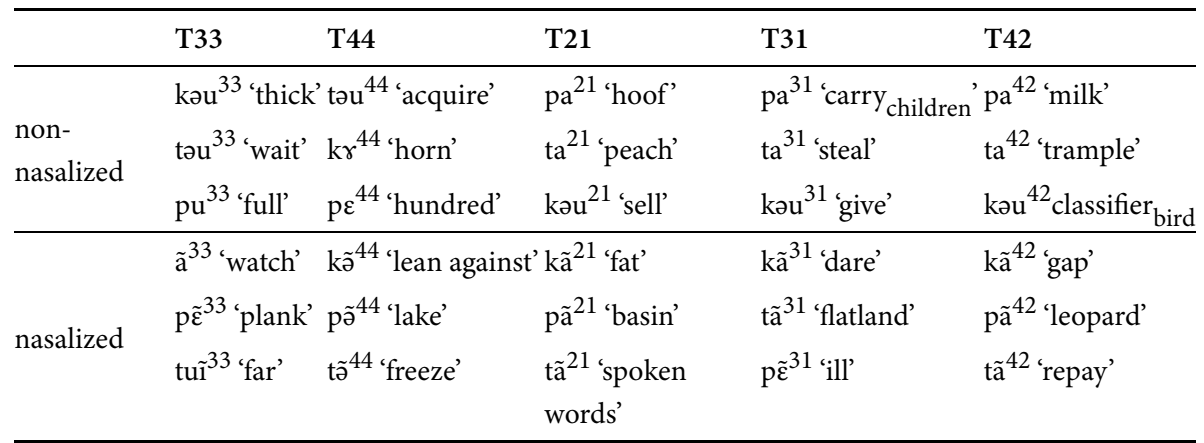

\subsubsection{Jinhua Bai}

Wang (2015) collected the data from four Bai speakers of Jinhua Village, Jianchuan County, including two males and two females.

Table 4. The speakers of Jinhua Bai

\begin{tabular}{llcllc}
\hline Name & No. & Age & Name & No. & Age \\
\hline YSN & F1 & 65 & YZH & M1 & 55 \\
YFK & F2 & 78 & YFS & M2 & 37 \\
\hline
\end{tabular}

Jinhua Bai has eight tones, and four of them are tense tones. Accordingly, there are four tense/lax pairs to be examined, i.e. T54-T55, T44-T33, T21-T31, and T42-T31. ${ }^{1}$ The recording samples that meet the needs of this paper in Wang (2015) is shown in Table 5. ${ }^{2}$

1. We follow here Wang (2015) who transcribes the tense tone T55 in Xu \& Zhao (1984) as T54.

2. Due to some uncontrollable factors in the recording process, the recording effects of individual syllables in different speakers are different. As for T44 and T21, the signals of ta44 and pã21 are more stable in males, while that of ka44 and mã21 are more stable in females. The relatively stable signals are selected for analysis for different gender speakers. 
Table 5. Recording samples

\begin{tabular}{|c|c|c|c|}
\hline Tone & Tone quality & Nasalized & Non-nasalized \\
\hline T44 & Tense & kã 44 'hurt by rocks in shoes' & ta $^{44}$ 'build ${ }_{\text {a bridge }}$ '/ka44 'fish scale' \\
\hline $\mathrm{T} 33$ & Lax & kã ${ }^{33}$ 'reduce' & $\mathrm{pa}^{33}$ 'foam' \\
\hline T54 & Tense & ts $^{\mathrm{h}} \tilde{\mathrm{o}}^{54}$ 'pungent' & $\mathrm{ta}^{54} \mathrm{ko}^{33}$ 'eldest brother' \\
\hline T55 & Lax & ts $^{\text {h }} \tilde{o}^{55}$ 'chaff' & $\mathrm{ta}^{55} \mathrm{ta}^{55}$ 'uncle' \\
\hline $\mathrm{T} 21$ & Tense & mã ${ }^{21}$ 'fur'/pã ${ }^{21}$ 'hoof' & $\mathrm{ta}^{21}$ 'peach' \\
\hline $\mathrm{T} 31$ & Lax & $\mathrm{kã}^{31}$ 'roll ${ }_{\text {the dough }}$ ' & $\mathrm{ta}^{31}$ 'steal' \\
\hline $\mathrm{T} 42$ & Tense & kã ${ }^{42}$ 'beat' & $\mathrm{ka}^{42}$ 'price' \\
\hline
\end{tabular}

\subsection{Sample recordings}

A number of research methods are applied in studying phonation, and each has its own advantages and disadvantages (Kong 2001:23-52). This paper investigates the phonation relying on Electroglottography (EGG) signals, as reliable parameters to describe the movements of vocal folds can be obtained without any intrusion (Kong 2001).

Cooledit 2.0 is used to record sounds on the left channel and the Electroglottograph Model 7050A is used to record EGG signals on the right channel. Two signals entered the computer through the mixer (XENYX 302 USB) and sound card (SBX). The sample rate was set at $22050 \mathrm{~Hz}$.

VoiceLab, implemented in Matlab, written by Phonetic Laboratory of Department of Chinese language and literature, Peking University, was applied to extract the F0, OQ, and SQ. A typical EGG signal is shown in Figure 1.

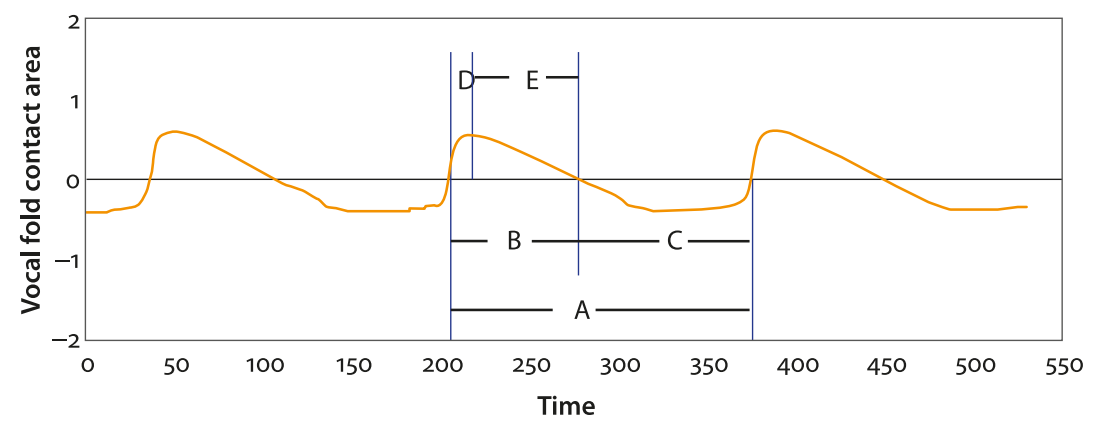

Figure 1. EGG signal

Phase A spans a period of the vibration of vocal folds. Phase B is the closed phase. Phase $\mathrm{C}$ is the open phase. Phase $\mathrm{D}$ is the closing phase. Phase $\mathrm{E}$ is the opening 
phase. Three parameters - fundamental frequency (F0), open quotient (OQ), and speed quotient (SQ) - are defined as follows (Kong, 2001).

$\mathrm{F} 0(\mathrm{~Hz})=1 /$ Period $(\mathrm{A})(\mathrm{s})$

$\mathrm{OQ}=$ Open phase $(\mathrm{C}) /$ period $(\mathrm{A}) \times 100 \%$

$\mathrm{SQ}=$ Opening phase $(\mathrm{E}) /$ Closing phase $(\mathrm{D}) \times 100 \%$

\subsection{Extraction of parameters}

According to the definition of F0, OQ, and SQ, the key to extracting these parameters is to find the closing point (the left boundary point of phase B), the opening point (the right boundary point of phase $B$ ), and the maximum value of the EGG pulse. The Voicelab provides us with two methods to extract parameters: differential method and criterion method. Previous studies show that phonation manner plays a role in tone distinction in Bai (Wang 2015; Li \& Wang 2016; Li 2017; Liu et al. 2019). Because the differential method is only suitable for the analysis of tones without special phonation features, only the criterion method is feasible in this study to extract the F0, OQ, and SQ. The criterion $25 \%$ is used in the extraction of all data.

Additionally, the parameters extracted in this study were time-normalized, that is, the F0, OQ, and SQ of all samples are composed of 20 points of data respectively. After the time-normalization, the first two data points and the last two points of each syllable were deleted, in that only the signals in the middle part are stable and reflect the phonetic features of the syllables. Ultimately, for each parameter, 16 stable data points were analyzed, which ensures the accuracy of this study.

\section{The data of Chengbei Bai}

Before the data analysis, the $t$-test is applied to examine whether there is a significant difference between tense and lax tones in terms of each parameter. The defined significance level is 0.05 . There is a significant difference between tense and lax tones, when $p<0.05$, otherwise, there is no significant difference. If the parameter shows a significant difference between tense and lax tones, its data points will be drawn in line charts to present overall trends.

In most situations, for the same parameter, the lines of two tones in a tense/ lax pair have the same trend; for example, Figure 2a shows two roughly parallel pitch lines of nasalized T44 and T33 of F1 (No. of speakers). The lines are distinctive in most cases, but sometimes they may be very similar, such as lines shown 
in Figure 2b. Based on Figure 2b, the pitch of T21 and T31 seems very similar, but the $t$-test result is significant, i.e. $p$-value $<0.05$. In such cases, the pitch of tense and lax tones are considered to be different, whereas it is unclear whether such a subtle difference may affect the perception of tones. There are very few cases where parameters between tense and lax tones are obviously distinct, but they have opposite trends as shown in Figure 2c. Further research is required to explore the reasons for uncommon cases. However, the cases, as shown in Figure $2 \mathrm{~b}$ or $2 \mathrm{c}$, just account for a very small percentage of the total.

\subsection{Phonation pattern}

Based on the result of the $t$-test and visual observation, a " +- " is entered when the parameters have no significant difference, a " + " is entered when the value of tense tone is greater than that of lax tone, and a "-" is entered when the value of tense tone is smaller. Tables 6 and 7 show the comparison results of tense and lax tones in each parameter for all speakers.

Table 6. Comparison results of tense and lax tones (nasalized)

\begin{tabular}{|c|c|c|c|c|c|c|c|c|c|c|}
\hline \multirow[b]{2}{*}{ Nasalized } & & \multicolumn{3}{|c|}{$\mathrm{T} 21 / \mathrm{T} 31$} & \multicolumn{3}{|c|}{ T42/T31 } & \multicolumn{3}{|c|}{ T44/T33 } \\
\hline & & F0 & OQ & SQ & F0 & OQ & SQ & F0 & OQ & SQ \\
\hline $\mathrm{F} 1$ & 16 & + & - & +- & + & + & - & + & + & + \\
\hline $\mathrm{F} 2$ & 20 & - & - & +- & + & - & - & + & - & - \\
\hline F3 & 50 & - & - & + & + & - & + & + & - & - \\
\hline $\mathrm{F} 4$ & 53 & + & - & +- & + & - & + & + & +- & +- \\
\hline M1 & 28 & - & - & + & + & - & - & + & + & +- \\
\hline M2 & 37 & +- & + & +- & + & - & + & + & + & +- \\
\hline M3 & 42 & - & +- & - & + & - & + & + & + & - \\
\hline M4 & 48 & - & - & + & + & - & - & + & - & - \\
\hline
\end{tabular}

The two tables above show that the realization of the distinction between tense and lax tones varies across individuals. The contrast between tense and lax tones is only reflected on one parameter for some speakers, while it is reflected on more than one parameter for others. To be specific, when nasalized, the distinction between T44 and T33 of F4 is only reflected on F0, and that between T21 and T31 of M2 is only reflected on OQ. Except for these two cases, the contrast between tense and lax tones is not only reflected on F0 but also on OQ or SQ. When non-nasalized, except for when the distinction between tense/lax tones is only reflected on F0 found in T21-T31 of F1, T44-T33 of F3, and T21-T31 of 


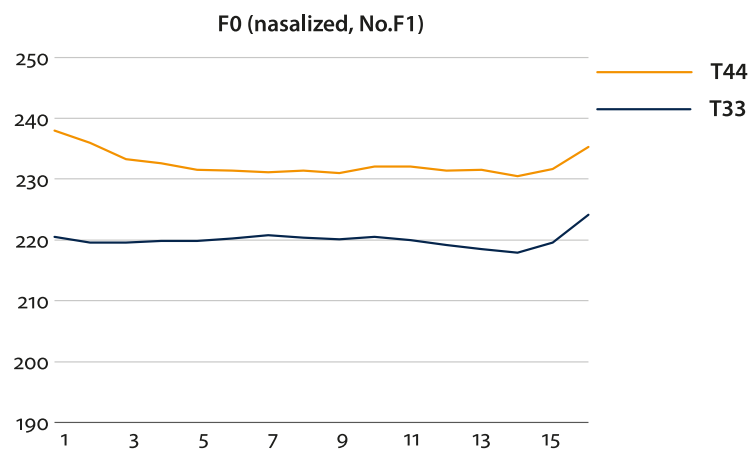

a.

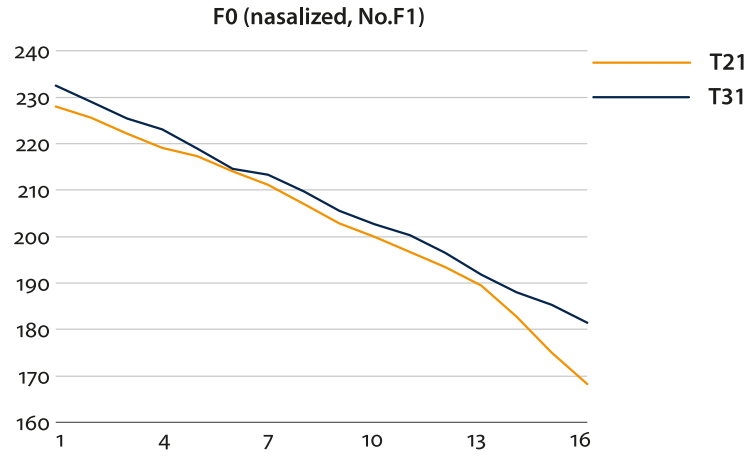

b.



c.

Figure 2. Examples of line charts ${ }^{3}$

3. The data points for all syllables are 16. That the maximum value of the horizontal axis of Figures A and C is 15 rather than 16 is caused by the representation method of line charts in EXCEL, not due to a lack of data points. 
Table 7. Comparison results of tense and lax tones (non-nasalized)

\begin{tabular}{|c|c|c|c|c|c|c|c|c|c|c|}
\hline \multirow{2}{*}{\multicolumn{2}{|c|}{ Non-nasalized }} & \multicolumn{3}{|c|}{$\mathrm{T} 21 / \mathrm{T} 31$} & \multicolumn{3}{|c|}{$\mathrm{T} 42 / \mathrm{T} 31$} & \multicolumn{3}{|c|}{$\mathrm{T} 44 / \mathrm{T} 33$} \\
\hline & & \multirow{2}{*}{$\frac{\text { F0 }}{+}$} & \multirow{2}{*}{$\frac{\mathrm{OQ}}{+-}$} & \multirow{2}{*}{$\frac{S Q}{+-}$} & \multirow{2}{*}{$\begin{array}{c}\text { F0 } \\
+\end{array}$} & \multirow{2}{*}{$\frac{\text { OQ }}{+}$} & \multirow{2}{*}{$\frac{\text { SQ }}{+-}$} & \multirow{2}{*}{$\frac{\text { F0 }}{+}$} & \multirow{2}{*}{$\frac{\text { OQ }}{-}$} & \multirow{2}{*}{$\frac{S Q}{+}$} \\
\hline F1 & 16 & & & & & & & & & \\
\hline F2 & 20 & + & - & +- & + & - & +- & + & + & + \\
\hline F3 & 50 & - & - & +- & + & - & + & + & - & + \\
\hline $\mathrm{F} 4$ & 53 & - & - & + & + & - & - & + & +- & +- \\
\hline M1 & 28 & - & +- & +- & + & - & - & + & - & + \\
\hline M2 & 37 & - & + & +- & + & - & + & + & +- & + \\
\hline M3 & 42 & - & - & +- & + & - & + & + & - & + \\
\hline M4 & 48 & - & - & + & + & + & +- & + & + & +- \\
\hline
\end{tabular}

M1, all the other tense/lax tone pairs are distinguished by more than one parameter. In total, there are three pairs of tense/lax tones for every speaker in nasalization or non-nasalization, thus, forty-eight sets of parameters between tense/lax tones is compared and examined. In 10\% (5/48) of cases, the distinction between tense and lax tones is only reflected on F0, while in the other $90 \%$ of cases the tense and lax tones do not only have contrast in F0 but also in phonation manner (OQ and SQ).

Regardless of the number of repetitions, as long as a set of the numerical relationship of three parameters illustrated by the labels ' $+/-/+-$ ' occurs and differs from others, it is treated as an independent phonation pattern (Kong 2001:289). Tables 8 and 9 show all the phonation patterns appearing in Chengbei Bai.

Table 8. Phonation patterns of Chengbei Bai (T21-T31)

\begin{tabular}{lccccccc}
\hline & \multicolumn{3}{c}{ Phonation pattern } & & \multicolumn{3}{c}{ Frequency } \\
\cline { 2 - 4 } \cline { 7 - 8 } No. & F0 & OQ & SQ & & Non-nasalized & Nasalized \\
\hline A1 & + & +- & +- & & 1 & \\
A2 & + & - & +- & & 1 & 2 \\
A3 & - & - & +- & & & 2 & 1 \\
A4 & - & - & + & & & 2 & 3 \\
A5 & - & +- & +- & & & 1 & \\
A6 & - & + & +- & & & 1 & 1 \\
A7 & +- & + & +- & & & & 1 \\
A8 & - & +- & - & & & & \\
\hline
\end{tabular}


Table 9. Phonation patterns of Chengbei Bai (T42-T31, T44-T33)

\begin{tabular}{|c|c|c|c|c|c|c|c|}
\hline \multirow[b]{3}{*}{ No. } & \multicolumn{3}{|c|}{ Phonation pattern } & \multicolumn{4}{|c|}{ Frequency } \\
\hline & \multirow[b]{2}{*}{ F0 } & \multirow[b]{2}{*}{ OQ } & \multirow[b]{2}{*}{ SQ } & \multicolumn{2}{|c|}{$\mathrm{T} 42-\mathrm{T} 31$} & \multicolumn{2}{|c|}{ T44-T33 } \\
\hline & & & & Non-nasalized & Nasalized & Non-nasalized & Nasalized \\
\hline $\mathrm{B} 1$ & + & + & - & & 1 & & 1 \\
\hline B2 & + & - & - & 2 & 3 & & 3 \\
\hline B3 & + & - & + & 3 & 4 & 4 & \\
\hline B4 & + & + & + & & & 1 & 1 \\
\hline B5 & + & +- & +- & & & 1 & 1 \\
\hline B6 & + & + & +- & 2 & & 1 & 2 \\
\hline B7 & + & - & +- & 1 & & & \\
\hline B8 & + & +- & + & & & 1 & \\
\hline
\end{tabular}

From Tables 8 and 9, a large variation in phonation is observed among different speakers of Chengbei Bai, because there are no dominant phonation patterns.

T21-T31: In nasalized syllables, there occur five different phonation patterns, and three of them (A2, A3, and A4) occur in non-nasalized syllables as well. The most frequent pattern is A4 that indicates a lower F0, a lower OQ, and a higher SQ of tense tones, occurring three times in total. In non-nasalized syllables, there occur six different phonation patterns, and two of them (A3 and A4) are relatively frequent, each of them occurring two times. The similarity between $\mathrm{A} 3$ and A4 is that F0 and OQ of tense tones are lower, but SQ is not lower than the lax tones. In short, the distribution of phonation patterns of T21-T31 bears no salient difference between nasalization and nonnasalization. By comparison, patterns $\mathrm{A} 2, \mathrm{~A} 3$, and $\mathrm{A} 4$ are more frequent than the other patterns, and their similarity lies in a lower OQ and a high SQ of tense tones.

T42-T31: In nasalized syllables, there are only three phonation patterns. B2 occurs three times, B3 occurs four times, and B1 occurs once. In nonnasalized syllables, there are four phonation patterns. As in the case of nasalization, B2 and B3 are most frequent. B2 occurs two times and $\mathrm{B} 3$ occurs three times. $\mathrm{B} 2$ and $\mathrm{B} 3$ are the same in a higher F0 and lower OQ of the tense tone but differ in SQ (B2 indicates that the tense tone has a lower SQ, but B3 indicates a higher SQ.). To put it simply, both in nasalization and non-nasalization, the most frequent phonation patterns in $\mathrm{T} 42-\mathrm{T} 31$ are $\mathrm{B} 2$ and $\mathrm{B} 3$, which suggests that $\mathrm{F} 0$ of tense tones is higher, OQ is lower, but SQ is higher or lower. 
T44-T33: In nasalized syllables, there are five phonation patterns in total. B2, which means that F0 of the tense tones is higher, but OQ and SQ are lower, occurs three times. B6, which means that both F0 and OQ of the tense tones are higher, but SQ is similar to that of the lax tones, occurs twice. In non-nasalized syllables, there are five different phonation patterns as well, but the most frequent pattern is neither B2 nor B6 but B3, occurring four times. B3 denotes that F0 and SQ of the tense tone is higher, but $\mathrm{OQ}$ is lower. In brief, the distribution of phonation patterns differs between nasalized and non-nasalized syllables. Specifically, SQ of the tense tone, i.e. T44, is higher in nonnasalized syllables, while it is lower in nasalized syllables. A higher SQ suggests a faster speed of closing vocal folds, accordingly the vocal folds are easily closed but hard to open. In other words, the vocal folds are tenser in this case than in a normal state. On the contrary, a lower SQ implies that the vocal folds are easy to open but hard to be closed, i.e. they are lax.

On the whole, the distinction between tense and lax tones in Chengbei Bai is mainly reflected on F0 and OQ. T21 has a lower F0 and OQ than T31, while T42 and T44 have a higher F0 and lower OQ than T31 and T33. Moreover, by comparing the phonation patterns, differences between nasalized and non-nasalized syllables were observed: (1) the distribution of phonation patterns of T42-T31 in nasalization is concentrated in B2 and B3, which is not attested in other tense/ lax tones and non-nasalized T42-T31; and (2) in terms of T44-T33, the distribution of phonation patterns between nasalization and non-nasalization is different. The difference is mainly reflected on the tense tone's SQ, which is higher in nonnasalized tense syllables, but lower in nasalized syllables.

\subsection{The strategy of tone distinction}

In addition to phonation pattern, the relationship between phonation and nasalization can be examined from the perspective of the strategy of tone distinction. The strategy of tone distinction is also analyzed based on F0, OQ, and SQ, but it is concerned with whether the parameter is significantly different or not, regardless of specific numerical relationship that is essential in the analysis of the phonation pattern. The assumption behind this concept is that once a parameter of two tones is significantly different, it is believed to be effective in the distinction of these two tones. For example, in the analysis of parameters of a speaker, if F0 and OQ between tones A and B are significantly different, while the SQ is similar, then a preliminary judgment is that this speaker applies the strategy of distinguishing F0 
and $\mathrm{OQ}$ of tone $\mathrm{A}$ from that of tone $\mathrm{B}$ to differentiate these two tones. ${ }^{4}$ The strategies of tone distinction employed in Chengbei Bai are summarized in Tables 10 and 11 .

Table 10. The strategies of tone distinction in Chengbei Bai (nasalized)

\begin{tabular}{llccc}
\hline & & \multicolumn{3}{c}{ Frequency } \\
\cline { 3 - 5 } Strategies & T21-T31 & T42-T31 & T44-T33 \\
\hline E & F0 & & 1 \\
F & OQ & 1 & \\
B & F0+OQ & 3 & 2 \\
C & F0+SQ & 1 & \\
A & F0+OQ+SQ & 3 & 8 & 5 \\
\hline
\end{tabular}

Table 11. The strategies of tone distinction in Chengbei Bai (non-nasalized)

\begin{tabular}{llccc}
\hline & & \multicolumn{3}{c}{ Frequency } \\
\cline { 2 - 5 } Strategies & T21-T31 & T42-T31 & T44-T33 \\
\hline E & F0 & 2 & & 1 \\
B & F0+OQ & 4 & 3 & 1 \\
C & F0+SQ & & & 1 \\
A & F0+OQ+SQ & 2 & 5 & 5 \\
\hline
\end{tabular}

Based on Tables 10 and 11, there are five strategies of tone distinction occurring in Chengbei Bai, i.e. A. F0+OQ+SQ (28); B. F0+OQ (13); E. F0 (4); C. F0+SQ (2); F. OQ (1). ${ }^{5}$ The number in the parentheses following each strategy is their frequency. The distribution of the strategy of tone distinction is discussed in detail as below.

T42-T31: In nasalized syllables, all speakers employ strategy A to distinguish T42 from T31. In non-nasalized syllables, to differentiate between T42 and T31, five speakers (62.5\%) apply strategy A, and another three $(37.5 \%)$ apply strategy B.

4. Whether this assumption is supported by perception experiments is open to further discussion.

5. The numbering here is not continuous, in that some strategies occur in Jinhua Bai but not in Chengbei Bai. 
T44-T33: The distribution of the strategy of tone distinction in nasalization is very similar to that in non-nasalization. In either case, five speakers (62.5\%) take strategy A to distinguish T44 from T33. Furthermore, few speakers take strategy B, E, or C.

T21-T31: In nasalized syllables, to distinguish T21 from T31, three speakers (37.5\%) apply the strategy A, and another three speakers apply strategy B. One speaker uses the strategy $\mathrm{C}$, and another speaker takes strategy F. In non-nasalized syllables, four speakers (50\%) take strategy B. Two speakers take strategy A, and two speakers take strategy E.

In summary, five different strategies are employed to distinguish tense tones from lax tones in Chengbei Bai. Among them, strategy A and B are most frequently used, especially strategy A that occurs twenty-eight times (58\%), while strategy $\mathrm{E}, \mathrm{C}$, and $\mathrm{F}$ are less frequent. In terms of the strategy of tone distinction, there are two aspects of differences between nasalized and non-nasalized syllables. First, there is only one strategy used in distinguishing nasalized T42 and T31, while it is not the case in non-nasalization. That is, the distinction between tense and lax tones is reflected along three parameters (F0, OQ, and SQ) when nasalized. Second, the least frequent strategies are strategy $\mathrm{E}(\mathrm{F} 0)$ and $\mathrm{F}(\mathrm{OQ})$, which are employed differently in nasalization and non-nasalization for distinguishing T21-T31. In non-nasalization, T21-T31 are distinguished by strategy E twice but never by the strategy F; on the contrary, in nasalization, T21-T31 are distinguished by strategy F once, but never by the strategy E. Based on the analysis of phonation pattern and strategy of tone distinction above, we shall further discuss the relationship between nasalization and phonation in the next section.

\subsection{Nasalization and phonation}

Through the analysis of the phonation pattern and the strategy of tone distinction, it is found that the phonetic basis of the distinction between tense and lax tones is different depending upon nasalization or non-nasalization. First, the distribution of phonation patterns of T44-T33 is distinct between in nasalized and nonnasalized syllables. SQ of T44 is lower than T33 when nasalized, while higher than T33 when non-nasalized. Second, the distribution of phonation patterns of nasalized T42-T31 is concentrated in pattern B2 and B3, and there is only one strategy of tone distinction (strategy A) corresponding to these two patterns. It means that both pitch (F0) and phonation manner are essential for the distinction of nasalized T42 and T31. Thirdly, there are two strategies that only one parameter is involved in tone distinction, i.e. strategy E (F0) and F (OQ). Nasal- 
ized T21-T31 can be distinguished by strategy $\mathrm{F}$ but not $\mathrm{E}$, while non-nasalized T21-T31 can be distinguished by strategy E but not $\mathrm{F}$.

These differences demonstrate that the effects of phonation in tone distinction is influenced by nasalization, and the way of influencing depends on the tone contour. First, the influence of nasalization on tenseness of the vocal folds is realized by movement on the soft palate.

In modal phonation, there is a negative correlation between $\mathrm{OQ}$ and F0, and a negative correlation between SQ and F0. In nasalized syllables of Chengbei Bai, the F0 of tense T44 is higher than that of lax tone T33, while the OQ and SQ of T44 are lower. That F0 is negatively correlated with both OQ and SQ represents a modal phonation of the vocal cords. However, in non-nasalized syllables, F0 is negatively correlated with OQ but positively correlated with $S Q$, which indicates a non-modal phonation of tense T44. As mentioned before ( $\$ 3.2), \mathrm{SQ}=$ Opening phase $(\mathrm{E}) /$ Closing phase $(\mathrm{D}) \times 100 \%$. Thus, a higher SQ implies that more time is required to open the vocal folds, i.e. vocal folds are hard to open but easily closed. Why is there such an increased tension of the vocal cords during non-nasalized?

It is assumed that the effect of nasalization on phonation is realized by lifting or lowering the soft palate. ${ }^{6}$ In Armstrong (1997)'s review, the well-known dentist Dr. Hudson Marken has pointed out that the function of the soft palate in phonation is modulating the tension of the vocal cords and the extent of resonance. The soft palate is directly jointed with the larynx, and the vocal folds are located within the larynx. When the soft palate is lifted or stretched, a pulling force will be applied to the back of the larynx. This pulling force combined with the movements of relative muscles can lead to a "high-tension state" of the vocal apparatus. The soft palate should be lifted in singing to get more resonance but cannot be lifted too much to make the vocal folds stiff and accordingly produce an unpleasant sound (Wu 2013). It seems that the higher the soft palate is raised, the tenser the vocal cords.

Lowering the soft palate allows part of the airstream to escape through the nose, leading to the nasalized timbre, and also relax the vocal folds. Conversely, in non-nasalization, the soft palate is lifted, and the vocal folds will be tightened. Both the research of phonation in singing and in nasalization confirms a positive correlation between the raising of the soft palate and the tension of the vocal folds.

6. Currently, the investigation concerning the effect of the movements of the soft palate on phonation is only found in the research of singing. The soft palate plays a very important role in singing. Some scholars believe that the position of the soft palate can be arbitrary in singing, but a majority of singing theories insist that the soft palate should be lifted to produce a more pleasant sound (Wu 2013). 
The higher the soft palate is raised, the tenser the vocal cords. The different states of the soft palate are corresponding to different tension of vocal folds.

Table 12. The relationship between movement of soft palate and tension of vocal folds

\begin{tabular}{lllll}
\hline & Improperly singing & Singing & Non-nasalization & Nasalization \\
\hline Soft palate & over raised & fully raised & raised & lowered \\
Tension of vocal folds & stiff & tense & slightly tense & lax \\
\hline
\end{tabular}

It is worth noting that the relationship between the soft palate and vocal cords illustrated in Table 12 is only found in level tones T44-T33, not in falling tones T21-T31 and T42-T31. It suggests that tone contours matter in the way that nasalization interacts with phonation. In the production of level tones, the frequency of vocal folds is relatively stable (that is why it is marked as a level tone.), accordingly there will be no significant change in its phonation manner. Therefore, the correlation between F0 and OQ or SQ can be compared with that represented in modal phonation or a certain type of non-modal phonation. However, the falling tone is not the case. In a falling tone, the frequency keeps falling (as indicated by its name). Along with a change in frequency, the phonation manner may also change, e.g. the end of falling tones is often accompanied by creaky voice. It suggests that the phonation parameters of falling tones (F0, OQ, and SQ) may be the result of more than one phonation type. Therefore, the correlation among them is not directly comparable to that represented in modal phonation or a certain type of non-modal phonation. A conclusion will be reached that the analysis of the correlation between different phonation parameters is only applicable to the level tones, e.g. T44-T33 in Chengbei Bai.

Furthermore, for falling tones, the effect of phonation manner in tone distinction is more obvious under nasalization.

As for T42-T31, the distribution of both the phonation pattern and the strategy of tone distinction implies that the distinction of T42 and T31 are reflected on three parameters (F0, OQ, and SQ) in nasalization, that is to say, phonation manner (OQ and SQ) plays an indispensable role in tone distinction, while such concentrated distribution is not found in non-nasalization. In other words, the effect of phonation manner is more obvious in the distinction of nasalized T42-T31.

In addition, in Chengbei Bai there are two strategies that distinguish between tense and lax tones with only one phonation parameter, i.e. strategy E (F0) and $\mathrm{F}(\mathrm{OQ})$. In nasalization, strategy $\mathrm{F}$ instead of strategy $\mathrm{E}$ is used to distinguish T21 from T31, which means that nasalized T21-T31 can be distinguished by only phonation manner (OQ), but cannot be by F0 alone. Conversely, in nonnasalized T21-T31, strategy E can be employed in tone distinction. It demon- 
strated that the effect of phonation manner is more obvious in the distinction of nasalized T21-T31. Combined with the evidence of T42-T31, we assume that for falling tones, the effect of phonation manner in tone distinction is more obvious in nasalized syllables.

To conclude, in Chengbei Bai, nasalization has an effect on phonation, but this effect varies with the types of tone contours. As for the level tones, the soft palate is raised higher in non-nasalization than in nasalization, thus the vocal folds of non-nasalized tones are tenser, and more likely to produce non-modal phonation, while for falling tones, the effect of phonation manner in tone distinction is more obvious in nasalized syllables. ${ }^{7}$

\section{The data of Jinhua Bai}

The method of extracting and analyzing data from Jinhua Bai is the same as that for Chengbei Bai. This section is a brief description of the relationship between phonation and nasalization in Jinhua Bai. The distribution of phonation patterns is summarized in Tables 13 and 14.

Table 13. Phonation patterns of Jinhua Bai (T44-T33, T54-T55)

\begin{tabular}{|c|c|c|c|c|c|c|c|}
\hline \multirow[b]{3}{*}{ No. } & \multicolumn{3}{|c|}{ Phonation pattern } & \multicolumn{4}{|c|}{ Frequency } \\
\hline & \multirow[b]{2}{*}{ F0 } & \multirow[b]{2}{*}{ OQ } & \multirow[b]{2}{*}{ SQ } & \multicolumn{2}{|c|}{ T44-T33 } & \multicolumn{2}{|c|}{ T54-T55 } \\
\hline & & & & Non-nasalized & Nasalized & Non-nasalized & Nasalized \\
\hline$\overline{\mathrm{A} 1}$ & + & - & + & & 2 & 2 & 1 \\
\hline A2 & + & - & - & & 1 & & \\
\hline A3 & + & - & +- & 1 & 1 & & 1 \\
\hline A4 & + & +- & - & 1 & & & \\
\hline A5 & - & - & - & 1 & & & \\
\hline A6 & +- & - & + & 1 & & & 1 \\
\hline A7 & +- & - & +- & & & 1 & \\
\hline A8 & +- & - & - & & & 1 & \\
\hline B1 & - & - & + & & & & 1 \\
\hline
\end{tabular}

7. It can be deduced from this conclusion that if the existing nasalization were to disappear in a language, the role of phonation manner in tone distinction would be more likely to remain in level tones. And this deduction seems to be supported by the data of Wuding Yi, in which the role of phonation manner in distinguishing tones is only reserved in level tones (Wang \& Kong 2009). However, further studies are needed to support such preservation due to the loss of nasalization. 
Table 14. Phonation patterns of Jinhua Bai (T21-T31, T41-T31)

\begin{tabular}{|c|c|c|c|c|c|c|c|}
\hline \multirow[b]{3}{*}{ No. } & \multicolumn{3}{|c|}{ Phonation pattern } & \multicolumn{4}{|c|}{ Frequency } \\
\hline & \multirow[b]{2}{*}{ F0 } & \multirow[b]{2}{*}{ OQ } & \multirow[b]{2}{*}{ SQ } & \multicolumn{2}{|c|}{ T21-T31 } & \multicolumn{2}{|c|}{ T42-T31 } \\
\hline & & & & Non-nasalized & Nasalized & Non-nasalized & Nasalized \\
\hline B1 & - & - & + & 1 & 1 & & \\
\hline B2 & - & +- & +- & 1 & 1 & & \\
\hline B3 & - & + & +- & & 1 & & \\
\hline B4 & +- & +- & + & & 1 & & \\
\hline B5 & - & - & +- & 1 & & & \\
\hline A1 & + & - & + & & & 1 & 1 \\
\hline $\mathrm{A} 2$ & + & - & - & 1 & & 1 & 1 \\
\hline A3 & + & - & +- & & & 1 & 2 \\
\hline A6 & +- & - & + & & & 1 & \\
\hline
\end{tabular}

Since the variation of phonation is salient even in four speakers, no significant difference of phonation pattern is found between nasalized and non-nasalized syllables according to Tables 13 and 14 .

As for the strategy of tone distinction, there are seven strategies applied in Jinhua Bai, i.e. A (F0+OQ+SQ); B (F0+OQ); C (F0+SQ); D (OQ+SQ); E (F0); F $(\mathrm{OQ})$; $\mathrm{G}(\mathrm{SQ})$. The distribution of different strategies in nasalization and nonnasalization is shown in Tables 15 and 16.

Table 15. Strategies of tone distinction in Jinhua Bai (nasalized)

\begin{tabular}{llcccc}
\hline & & \multicolumn{4}{c}{ Frequency } \\
\cline { 2 - 5 } Strategies & T21-T31 & T42-T31 & T44-T33 & T55-T54 \\
\hline E & F0 & 1 & & & \\
G & SQ & 1 & & & \\
B & F0+OQ & & 2 & 1 & 1 \\
A & F0+OQ+SQ & 2 & 2 & 3 & 3 \\
\hline
\end{tabular}

Table 16. Strategies of tone distinction in Jinhua Bai (non-nasalized)

\begin{tabular}{|c|c|c|c|c|c|}
\hline & & \multicolumn{4}{|c|}{ Frequency } \\
\hline \multicolumn{2}{|c|}{ Strategies } & $\mathrm{T} 21-\mathrm{T} 31$ & T42-T31 & T44-T33 & Т55-T54 \\
\hline $\mathrm{F}$ & OQ & & & & 1 \\
\hline $\mathrm{D}$ & $\mathrm{OQ}+\mathrm{SQ}$ & & 1 & 1 & 1 \\
\hline $\mathrm{E}$ & F0 & 1 & & & \\
\hline $\mathrm{C}$ & $\mathrm{F} 0+\mathrm{SQ}$ & & & 1 & \\
\hline B & $\mathrm{F} 0+\mathrm{OQ}$ & 1 & 1 & 1 & \\
\hline A & $\mathrm{F} 0+\mathrm{OQ}+\mathrm{SQ}$ & 2 & 2 & 1 & 2 \\
\hline
\end{tabular}


Based on Tables 15 and 16, for T21-31 and T41-T31, there is no obvious difference in the strategy of tone distinction between nasalization and nonnasalization. To be specific, in either nasalization or non-nasalization of T21-T31 and T42-T31, strategy A is applied four times in total, strategy B twice, and strategy $\mathrm{E}$ once. Moreover, in either case, tones can be distinguished solely by phonation manner, which is marked by the use of strategy without F0. Strategy G (SQ) is employed in nasalization and strategy $\mathrm{D}(\mathrm{OQ}+\mathrm{SQ})$ in non-nasalization.

As for the level tones, i.e. T44-T33 and T54-T55, differences were found between nasalized and non-nasalized cases. It was observed in Table 15 that the distribution of strategy of tone distinction is concentrated in strategies A and B. The shared distinctive parameters in these two strategies are F0 and OQ; that is to say, F0 and OQ are two basic features for tone distinction in nasalization. In nonnasalization, the distribution of strategy of tone distinction is relatively scattered, in that there occur five different strategies in total. Among the five strategies, three of them includes the F0 parameter (A, B, and C), and two of them excludes the F0 parameter (D and F). Strategy D and F, which indicate that tense and lax tones are distinguished only by phonation manner, occurred a total of three times, accounting for $37.5 \%$. In short, as for the distinction of level tones in Jinhua Bai, F0 may not contribute to the distinction of tones in non-nasalization, but this would not happen under nasalization, i.e. F0 is obligatory in distinguishing nasalized level tones. In other words, tense tones can be distinguished from lax tones by OQ and SQ, characterizing phonation manner, in non-nasalization, while it is not feasible in nasalization. This suggests that the effect of phonation manner is more salient in distinguishing non-nasalized tones.

The analysis results of Jinhua Bai further support findings in Chengbei Bai with regard to the level tones. Both of them demonstrate that the vocal folds are lax when the soft palate is lowered in nasalization, whereas they are tense when the soft palate is raised in non-nasalization. Tense vocal folds are more likely to produce non-modal phonation, which strengthens the effect of phonation manner in tone distinction.

It is worth noting that a difference in nasalization vs. non-nasalization is not seen in analyzing the strategies of distinguishing level tones in Chengbei Bai, but is observed in Jinhua Bai. It could be possible that such inconsistency reflects a variation of phonation in different dialects. Strategy $\mathrm{D}(\mathrm{OQ}+\mathrm{SQ})$, which can highlight a difference between nasalized and non-nasalized syllables, is not employed in Chengbei Bai, but is found in Jinhua Bai. 


\section{Conclusion}

The speech production mechanism as a whole involves four main components: airstream process, phonation process, oro-nasal process, and articulatory process (Ladefoged \& Johnson 2011:5). Different processes are relatively independent but also interact with each other in most cases. Matisoff (1975) pointed out that there exists a natural connection between nasalization and the articulation of laryngeal sounds, which he has dubbed rhinoglottophilia, while this study, by analyzing the tense and lax contrasts in the Bai language, reveals how nasalization influences phonation. Regarding the whole phonological system, nasalization will not undermine the existing contrasts between tense and lax tones, but in part, finetune the way that tense tones are distinguished from lax tones, and such finetuning possibly varies among individuals.

As far as level tones are concerned, whether in Chengbei or Jinhua Bai, the role of phonation manner in tone distinction is not evident in nasalization because tense tones can be distinguished from lax tones only by pitch. However, in non-nasalization, phonation manner plays an indispensable role in tone distinction, in the sense that the contrast between tense and lax tones are reflected not only on F0 but also on OQ and SQ. Moreover, non-nasalized tense tones are more likely to be accompanied by non-modal phonation that is characterized by a higher SQ. In terms of articulatory explanation, high SQ in non-modal phonation is the result of vibration of the tightened vocal folds, and tension of vocal folds is caused by raising the soft palate in non-nasalization. As for the falling tones, the role of phonation manner in tone distinction is more salient in nasalization than in non-nasalization in Chengbei Bai, but it is not attested in Jinhua Bai.

Based on intra-comparison, nasalization is a weakened form of the nasal endings ${ }^{*}-n$ and ${ }^{*}-\eta$ in proto-Bai (Wang 2012:65), and the role of phonation manner in tone distinction is stimulated by the devoicing of voiced obstruents (stops, affricates, and fricatives) and the loss of the non-nasal endings. Both nasalization and phonation can be distinctive features in languages, but in Bai, they are being gradually replaced by other phonetic features. It is known that the substitution of some inherited phonemes by innovative features will result in the reorganization of the phonological system, but such a process cannot be directly observed until it has been completed. However, the extraction and analysis of the phonation parameters, i.e. F0, OQ, and SQ in this study, can uncover the underlying phonation variations that have not yet been reflected in the phonology. 


\section{Acknowledgements}

This research reported herein was supported by the fund from Ministry of Education of China (No.19JJD740001). We thank our two anonymous reviewers for their valuable and detailed comments. We are extremely grateful to all Bai speakers who participated in this study and to a number of people who have generously helped us in the data collection. We also would like to thank Karl Holm (The Chinese University of Hong Kong), Qiong Wu (University of Hawaii System) and Yik-po Lai (The Chinese University of Hong Kong) for reading the pre-final version of the article and providing numerous helpful suggestions to its content and to the authors' English. Any remaining errors are ours.

\section{References}

Armstrong, WM.G. 1997. Changge shi de ruan'e [On the soft palate during singing]. In Liang Xue (ed.), Gechang de fangfa [Techniques of singsing], 2nd edn., 398-403. Beijing: Zhongguo Wenlian Chubanshe.

Bao, Huaiqiao \& Lü, Shinan. 1992. Mengguyu Chaha'er hua yuanyin songjin de shegnxue fenxi [The phonetic analysis of tense and lax vowels of Chaha'er Mongolian]. Minzu Yuwen [Minority Languages of China] 1992(1). 61-68

Benner, Allison. 2009. Production and perception of laryngeal constriction in the early vocalizations of Bai and English infants. Victoria: University of Victoria. (Doctoral dissertation.)

Chao, Yuen Ren. 1980[1930]. A system of "tone letters". Fangyan [Dialect] 1980(2). 81-83. (Originally published in Le Maître Phonétique 45(30). 24-27.)

Dai, Qingxia \& Zhao, Yanzhen. 2009. Zhaozhuang Bai yu gaikuang [A brief introduction of Bai language Zhaozhuang speech]. Minzu Yuwen [Minority languages of China] 2009(3).109-126.

Edmondson, Jerold A. \& Esling, John H. \& Li, Shaoni \& Harris, Jimmy G. \& Ziwo, Lama. 2000. Lun yiyu, baiyu de yinzhi he shaohuiyanjidai de guanxi: Houjing anli yanjiu [The aryepiglottic holds and voice quality in Yi and Bai languages: Laryngoscopic case studies] Minzu Yuwen [Minority languages of China] 2000(6). 47-53.

Edmondson, Jerold A. \& Li, Shaoni. 1994. Voice quality and voice quality change in the Bai language of Yunnan Province. Linguistics of the Tibeto-Burman Area 17(2). 49-68.

Edmondson, Jerold A. \& Ziwo, Lama \& Esling, John H. \& Harris, Jimmy G. \& Li, Shaoni. 2001. The aryepiglottic folds and voice quality in the Yi and Bai languages: Laryngoscopic case studies. Mon-Khmer Studies Journal 31. 83-100.

Esling, John H. 2005. There are no back vowels: The laryngeal articulator model. Canadian Journal of Linguistics 50(1-4). 13-44. https://doi.org/10.1353/cjl.2007.0007

Fant, Gunnar. 1960. Acoustic theory of speech production: With calculations based on X-ray studies of Russian articulations. The Hague: Mouton.

Garellek, Marc \& Ritchart, Amanda \& Kuang, Jianjing. 2016. Breathy voice during nasality: A cross-linguistic study. Journal of Phonetics. 59. 110-121. https://doi.org/10.1016/j.wocn.2016 .09 .001

Gong, Xijie \& Li, Xuan \& Wang, Feng. 2018. Dali Longfengcun Bai yu gaikuang [A field report on Longfeng Bai]. Hanzangyu Xuebao [Journal of Sino-Tibetan Linguistics] 10. 63-90. 
Kong, Jiangping. 1996. Hani yu fasheng leixing shengxue yanjiu ji yinzhi gainian de taolun [Discussion on the phonation type and sound quality in Hani language]. Minzu Yuwen [Minority Languages of China] 1996(1). 40-46.

Kong, Jiangping. 2001. Lun yuyan fasheng [On language phonation]. Beijing: China Minzu University Press.

Ladefoged, Peter \& Johnson, Keith. 2011. A course in phonetics. 6th edn. Boston: Cengage.

Li, Shaoni. 1992. Lun Bai yu de shegnmen hunhe jicayin [On the combination of glottal squeezing and frication in the Bai language]. Minzu Yuwen [Minority Languages of China] 1992(4). 68-72.

Li, Shaoni \& Ai, Jierui. 1990. Yunnan Jianchuan Bai yu yinzhi he yindiao leixing: Diannao yuyin shiyan baogao [The sound quality and tone type of Jianchuan Bai in Yunnan: A report of computer phonetics experiment]. Zhongyang Minzu Daxue Xuebao (Zhexue Shehui Kexue Ban) [Journal of Minzu University of China (Philosophy and Social Sciences Edition)] 1990(5). 70-74.

Li, Xuan. 2017. Meiba Bai yu shengdiao de fasheng bianyi yanjiu [A study of variations of phonation features in Meiba Bai]. Beijing: Peking University. (Master's thesis.)

Li, Xuan \& Wang, Feng. 2016. Meiba Baiyu shengdiao de fasheng bianyi chutan [A preliminary study of variations of phonation features in Meiba Bai]. Yuyanxue Luncong [Essays on Linguistics] 54. 179-196.

Liu, Wen \& Wang, Feng \& Kong, Jiangping. 2019. Beiwuliqiao Bai yu shengdiao de fasheng ji bianyi yanjiu [An acoustic study on the phonation variations of tones in Beiwuliqiao Bai]. Dangdai Yuyanxue [Contemporary Linguistics] 2019(1). 119-138.

Maddieson, Ian \& Ladefoged, Peter. 1985. "Tense" and "lax" in four minority languages of China. Journal of Phonetics 13(4). 433-454. https://doi.org/10.1016/Soo95-4470(19)30788-0

Matisoff, James A. 1975. Rhinoglottophilia: The mysterious connection between nasality and glottality. In Ferguson, Charles A. \& Hyman, Larry H. \& Ohala, John J. (eds.), Nasálfest: Papers from a symposium on nasals and nasalization, 265-287. Stanford: Language Universals Project, Department of Linguistics, Stanford University.

Ohala, John J. 1971. Monitoring soft palate movements in speech. Journal of the Acoustical Society of America 50 (1A). 140. (Paper presented at the 81st Meeting of the Acoustical Society of America, Washington, D.C, 20-23 April 1971.) https://doi.org/10.1121/1.1977664

Ohala, John J. 1972. Physical models in phonology. In Rigault, André \& Charbonneau, René (eds.), Proceedings of the 7th International Congress of Phonetic Sciences: Held at the University of Montreal and McGill University 22-28 August 1971, 1166-1171. The Hague: Mouton. https://doi.org/10.1515/9783110814750-167

Singhal, Nishant \& Das, Pradip K. 2013. Study of acoustic properties of nasal and nonnasal vowels in temporal domain. Computer Science and Information Technology 3(6). 305-314. https://doi.org/10.5121/csit.2013.3635

Wang, Feng. 2012. Yuyan jiechu yu yuyan bijiao: Yi Bai yu weili [Language contact and language comparison: The case of Bai]. Beijing: The Commercial Press.

Wang, Feng. 2015. Variations of laryngeal features in Jianchuan Bai, Journal of Chinese Linguistics 43 (1B). 434-452. https://doi.org/10.1353/jcl.2015.0033

Wang, Feng \& Kong, Jiangping. 2009. Wuding Yiyu songjinyin yanjiu [A study of lax/tense voice in the Wuding Yi]. Zhongguo Yuyanxue [Chinese linguistics] 2. 98-118.

Wu, Fan. 2013. Lun ruane yu gechang fasheng zhi guanxi [On the relationship between soft palate and phonation in singing]. Wuhan: Wuhan Conservatory of Music. (Master's thesis.) 
Xu, Lin \& Zhao, Yansun. 1984. Baiyu jianzhi [A brief description of Bai]. Beijing: The Ethnic Publishing House.

Yang, Xiaoxia \& Gao, Tianjun. 2016. Cong fashengtai kan Bai yu de jinyin [On tense sounds in the Bai language from in the perspective of phonation]. Minzu Yuwen [Minority Languages of China] 2016(6). 90-95.

Zhao, Jincan. 2010. Yunnan Heqing Bai yu yanjiu [A description of Heqing Bai language]. Beijing: Minzu University of China. (Doctoral dissertation.)

\section{Authors' addresses}

Feng Wang (corresponding author)

Center for Chinese Linguistics

Department of Chinese Language and Literature

Peking University

5 Yiheyuan Road

Beijing 100871

China

wfwf@pku.edu.cn

\section{Publication history}

Date received: 6 May 2019

Date accepted: 17 August 2019

Published online: 15 December 2021 